\title{
A SUB-SUPERSOLUTION METHOD FOR NONLINEAR ELLIPTIC SINGULAR SYSTEMS WITH NATURAL GROWTH AND SOME APPLICATIONS
}

\author{
JOSÉ CARMONA, PEDRO J. MARTÍNEZ-APARICIO, AND ANTONIO SUÁREZ
}

\begin{abstract}
In this paper we give a sub-supersolution method for nonlinear elliptic singular systems with quadratic gradient whose model system is the following

$$
\begin{cases}-\Delta u+v^{\beta} \frac{|\nabla u|^{2}}{u^{\alpha}}=f_{1}(x, u, v) & \text { in } \Omega, \\ -\Delta v+u^{\mu} \frac{|\nabla v|^{2}}{v^{\gamma}}=f_{2}(x, u, v) & \text { in } \Omega, \\ u=v=0 & \text { on } \partial \Omega,\end{cases}
$$

where $\Omega$ is a smooth bounded domain of $\mathbb{R}^{N}(N \geq 3), \beta, \mu \geq 0,0<\alpha, \gamma<1$ and regular $f_{1}, f_{2}$ functions. Moreover, we apply it to prove existence of solution for some systems, including the classical Lotka-Volterra models with gradient terms. Specifically, we study the competition and the symbiotic LoktaVolterra systems.
\end{abstract}

\section{INTRODUCTION}

The aim of this paper is to provide a sub-supersolution method for the following nonlinear elliptic singular system with natural growth

$$
\begin{cases}-\Delta u+g_{1}(v) \frac{|\nabla u|^{2}}{u^{\alpha}}=f_{1}(x, u, v) & \text { in } \Omega, \\ -\Delta v+g_{2}(u) \frac{|\nabla v|^{2}}{v^{\gamma}}=f_{2}(x, u, v) & \text { in } \Omega \\ u=v=0 & \text { on } \partial \Omega\end{cases}
$$

where $\Omega$ is a smooth bounded domain of $\mathbb{R}^{N}(N \geq 3)$ the functions $g_{1}, g_{2} \in$ $C([0,+\infty))$ and $f_{1}, f_{2} \in C(\bar{\Omega} \times[0,+\infty) \times[0,+\infty))$ verifying some general conditions detailed below.

Regarding the literature there are several papers about equations with quadratic gradient terms. The existence of solutions of the equation

$$
-\Delta u+g(u)|\nabla u|^{2}=a(x) \quad \text { in } \Omega, \quad u=0 \quad \text { on } \partial \Omega,
$$

for every function $a(x)$ in a given Lebesgue space has been systematically studied in $[4, \underline{5,} \underline{6]}$ and references therein (in fact, for a more general nonlinear term $H(x, u, \nabla u)$ instead of $\left.g(u)|\nabla u|^{2}\right)$. They consider in the lower order term a continuous $g$ in $\mathbb{R}$ which does not satisfy any growth restriction and the sign condition $g(s) s \geq 0$ for every $s \in \mathbb{R}$ is assumed. Thanks to the presence of the lower order term the Dirichlet problem associated to the equation is allowed to have finite energy weak solutions. 
In $[15]$ and $[7]$ some of the above results were extended to the case of systems.

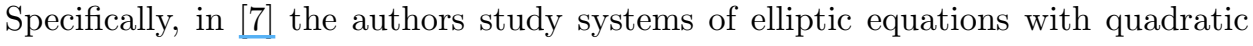
gradient. They consider a general system

$$
-\Delta u_{i}+H_{i}(x, u, \nabla u)=a_{i}(x) \quad \text { in } \Omega, \quad u=0 \quad \text { on } \partial \Omega, \quad i=1, \ldots, n
$$

where $u=\left(u_{1}, \ldots, u_{n}\right), a_{i} \in H^{-1}(\Omega)$ and the quadratic terms $H_{i}(x, u, \nabla u)$ satisfy a more general one-side condition than the sign condition, but in the case $H_{i}(x, u, \nabla u)=g_{i}(u)|\nabla u|^{2}$ this one-side hypothesis is equivalent to the sign condition. In their case $g_{i}$ is continuous in $\mathbb{R}^{n}$ and they prove the existence of solution in the Sobolev space.

In the last years, equation (1.2) has attracted much attention by the presence of singular terms in front of the gradient, see $[1,2,8]$ and references therein.

In $\underline{[11]}$ we prove that a sub-supersolution method works for equations of the form

$$
-\Delta u+\frac{|\nabla u|^{2}}{u^{\alpha}}=f(\lambda, u)
$$

and we apply it to different models.

In this paper we focus our attention in systems with quadratic gradient and singular terms as (1.1).

Let us mention that the sub-supersolution method is valid for semilinear systems,

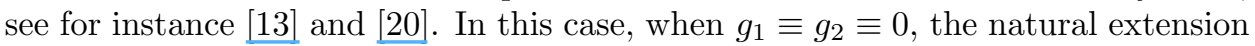
of the scalar definition of sub-supersolution depends on the monotonicity of the functions $f_{1}$ and $f_{2}$ with respect to $v$ and $u$, respectively. A general definition was given in $\underline{[13]}$ and $\underline{[20]}$ where a pair of functions $(\underline{u}, \underline{v}),(\bar{u}, \bar{v}), \underline{u}, \bar{u}, \underline{v}, \bar{v} \in H^{1}(\Omega) \cap$ $L^{\infty}(\Omega)$ is called a sub-supersolution if

$$
\begin{aligned}
& \underline{u} \leq \bar{u}, \quad \underline{v} \leq \bar{v} \quad \text { in } \Omega, \\
& \underline{u} \leq 0 \leq \bar{u}, \quad \underline{v} \leq 0 \leq \bar{v} \quad \text { on } \partial \Omega \text {, }
\end{aligned}
$$

and

$$
\begin{array}{ll}
-\Delta \underline{u} \leq f_{1}(x, \underline{u}, v), & -\Delta \bar{u} \geq f_{1}(x, \bar{u}, v), \quad \forall v \in[\underline{v}, \bar{v}], \\
-\Delta \underline{v} \leq f_{2}(x, u, \underline{v}), & -\Delta \bar{v} \geq f_{2}(x, u, \bar{v}), \quad \forall u \in[\underline{u}, \bar{u}],
\end{array}
$$

where, given two ordered functions $z \leq w$, we have denoted

$$
[z, w]:=\left\{q \in L^{\infty}(\Omega): z(x) \leq q(x) \leq w(x)\right\}
$$

(see also [19] where it is proved the validity of the method for singular semilinear systems). Assuming the existence of a sub-supersolution, $(\underline{u}, \underline{v}),(\bar{u}, \bar{v})$, there exists a solution $(u, v) \in I \equiv[\underline{u}, \bar{u}] \times[\underline{v}, \bar{v}]$ of the semilinear system (i.e. (1.1) with $\left.g_{1} \equiv g_{2} \equiv 0\right)$.

When the reaction terms depend on the gradient, i.e.,

$$
-\Delta u=f_{1}(x, u, v, \nabla u, \nabla v), \quad-\Delta v=f_{2}(x, u, v, \nabla u, \nabla v)
$$

and the functions $f_{1}$ and $f_{2}$ are regular verifying some hypotheses, the definition is $(\operatorname{see} \underline{[21]})$

$$
\begin{array}{lll}
-\Delta \underline{u} \leq f_{1}(x, \underline{u}, v, \nabla \underline{u}, \nabla v), & -\Delta \bar{u} \geq f_{1}(x, \bar{u}, v, \nabla \bar{u}, \nabla v), & \forall v \in[\underline{v}, \bar{v}], \\
-\Delta \underline{v} \leq f_{2}(x, u, \underline{v}, \nabla u, \nabla \underline{v}), & -\Delta \bar{v} \geq f_{2}(x, u, \bar{v}, \nabla u, \nabla \bar{v}), & \forall u \in[\underline{u}, \bar{u}] .
\end{array}
$$

Assuming again the existence of a sub-supersolution, the existence of a solution $(u, v) \in I$ follows. 
In this paper, we use the above definition of sub-supersolution, and taking advantage of the form of equation, we overcome the singularities difficulty of the system (1.1) with respect to $u$ and $v$. Hence, for our system (1.1) we define a couple of sub-super solution as follows

$$
\begin{array}{ll}
-\Delta \underline{u}+g_{1}(v) \frac{|\nabla \underline{u}|^{2}}{\underline{u}^{\alpha}} \leq f_{1}(x, \underline{u}, v), \quad-\Delta \bar{u}+g_{1}(v) \frac{|\nabla \bar{u}|^{2}}{\bar{u}^{\alpha}} \geq f_{1}(x, \bar{u}, v), \quad \forall v \in[\underline{v}, \bar{v}], \\
-\Delta \underline{v}+g_{2}(u) \frac{|\nabla \underline{v}|^{2}}{\underline{v}^{\gamma}} \leq f_{2}(x, u, \underline{v}), \quad-\Delta \bar{v}+g_{2}(u) \frac{|\nabla \bar{v}|^{2}}{\bar{v}^{\gamma}} \geq f_{2}(x, u, \bar{v}), \quad \forall u \in[\underline{u}, \bar{u}] .
\end{array}
$$

Moreover, we apply this method to prove existence of positive solution for some systems, including the classical Lotka-Volterra models confronted with the Laplacian operator perturbed by a singular gradient term, that is, the following systems

$$
\begin{cases}-\Delta u+g_{1}(v) \frac{|\nabla u|^{2}}{u^{\alpha}}=u(\lambda-u-b v) & \text { in } \Omega, \\ -\Delta v+g_{2}(u) \frac{|\nabla v|^{2}}{v^{\gamma}}=v(\mu-v-c u) & \text { in } \Omega, \\ u=v=0 & \text { on } \partial \Omega,\end{cases}
$$

where $\lambda, \mu \in \mathbb{R}$ and $b \cdot c>0$. Here, $u(x)$ and $v(x)$ denote the densities of two species, $\lambda$ and $\mu$ represent the growth rates of the species, $b$ and $c$ measure the interaction rates between both species; if $b, c>0$ they are competing and if $b, c<0$ cooperating. Moreover, in (1.4) a nonlinear convective term is included with a singular term. This term is accompanied by a nonlinear function depending on the other species. We give conditions on $\lambda$ and $\mu$ that assure the existence of a coexistence state of (1.4), that is, a solution with both components positive.

The structure of the article is: in Section 2 we study an auxiliary scalar equation that we use in Section 3 to prove the validity of the sub-supersolution method for (1.1). Section 4 is devoted to applications of the method.

\section{AN AUXILIARY SCALAR EQUATION}

Frizzing one of the unknown in each equation of (1.1) we are led to consider the scalar boundary value problem

$$
\begin{cases}-\Delta w+m(x) \frac{|\nabla w|^{2}}{w^{\theta}}=f(x, w) & \text { in } \Omega, \\ w=0 & \text { on } \partial \Omega,\end{cases}
$$

for convenient functions $m$ and $f$ and a parameter $0<\theta<1$. In order to use monotone methods for (2.1), as was pointed out in $[11]$ for $m(x)$ constant, it is useful to consider a positive function $g \in C(0,+\infty)$ such that, denoting $G(u)=\int_{1}^{u} g(s) d s$, the function $e^{-G(s)}$ belongs to $L^{1}(0,1)$ and we define also $\Psi$ by

$$
\Psi(s):=\int_{0}^{s} e^{-G(t)} d t, \quad s>0 .
$$

Observe that if there exists $M \geq 0$ such that $f(x, s)+M s$ is nondecreasing for a.e. $x \in \Omega$ then $f(x, s)+M \Psi(s) e^{G(s)}$ is also nondecreasing for a.e. $x \in \Omega$. Thus, adding the term $M \Psi(w) e^{G(w)}$ in (2.1) it becomes

$$
\begin{cases}-\Delta w+m(x) \frac{|\nabla w|^{2}}{w^{\theta}}+M \Psi(w) e^{G(w)}=f(x, w)+M \Psi(w) e^{G(w)} & \text { in } \Omega \\ w=0 & \text { on } \partial \Omega .\end{cases}
$$


Therefore, in order to study (2.1) using sub-supersolution, we need to establish a comparison principle for the problem

$$
\begin{cases}-\Delta w+m(x) \frac{|\nabla w|^{2}}{w^{\theta}}+M \Psi(w) e^{G(w)}=f_{0}(x) & \text { in } \Omega, \\ w=0 & \text { on } \partial \Omega,\end{cases}
$$

where $0<m(x) \in L^{\infty}(\Omega), 0<\theta<1$ and $0 \leq f_{0}(x) \in L^{2 N /(N+2)}(\Omega), f_{0} \not \equiv 0$. Observe that this problem is similar to that studied in [11] but here the function $g$, from which are defined $G$ and $\Psi$, is arbitrary and non necessary related with the gradiend lower order term. When $M=0$, that is,

$$
\begin{cases}-\Delta w+m(x) \frac{|\nabla w|^{2}}{w^{\theta}}=f_{0}(x) & \text { in } \Omega \\ u=0 & \text { on } \partial \Omega\end{cases}
$$

this problem has solution (see [8]) and it is unique (see [3]).

The concept of sub and super-solution for the problem (2.2) is the following:

Definition 2.1. A sub-solution of $(2.2)$ is a function $\underline{u} \in H_{0}^{1}(\Omega)$ such that $0<\underline{u}$ a.e. in $\Omega, \frac{|\nabla \underline{u}|^{2}}{\underline{u}^{\gamma}}, \Psi(\underline{u}) e^{G(\underline{u})} \in L^{1}(\Omega)$ and for every $\phi \in H_{0}^{1}(\Omega) \cap L^{\infty}(\Omega), \phi \geq 0$,

$$
\int_{\Omega} \nabla \underline{u} \cdot \nabla \phi+\int_{\Omega} m(x) \frac{|\nabla \underline{u}|^{2}}{\underline{u}^{\theta}} \phi+M \int_{\Omega} \Psi(\underline{u}) e^{G(\underline{u})} \phi \leq \int_{\Omega} f_{0}(x) \phi .
$$

Similarly $\bar{u} \in H^{1}(\Omega)$ such that $0<\bar{u}$ a.e. in $\Omega, \frac{|\nabla \bar{u}|^{2}}{\bar{u}^{\theta}}, \Psi(\bar{u}) e^{G(\bar{u})} \in L^{1}(\Omega)$ and for every $\phi \in H_{0}^{1}(\Omega) \cap L^{\infty}(\Omega), \phi \geq 0$,

$$
\int_{\Omega} \nabla \bar{u} \cdot \nabla \phi+\int_{\Omega} m(x) \frac{|\nabla \bar{u}|^{2}}{\bar{u}^{\theta}} \phi+M \int_{\Omega} \Psi(\bar{u}) e^{G(\bar{u})} \phi \geq \int_{\Omega} f_{0}(x) \phi,
$$

is called a super-solution of (2.2). We say that $u \in H_{0}^{1}(\Omega)$ is a solution of (2.2) if it is a sub and super-solution of (2.2).

We recall some classical results about the regularity of the equation (2.2) with $f_{0}(x)=f(x, w(x))$, that is the non-linear equation

$$
\begin{cases}-\Delta w+m(x) \frac{|\nabla w|^{2}}{w^{\theta}}+M \Psi(w) e^{G(w)}=f(x, w) & \text { in } \Omega, \\ w=0 & \text { on } \partial \Omega .\end{cases}
$$

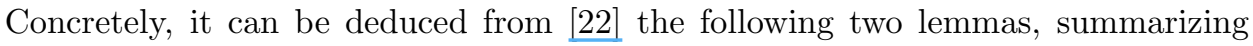
some known $L^{\infty}(\Omega)$-estimates for sub-solutions of (2.3). The first one deals with a subcritical function $f$, here the $L^{\infty}(\Omega)$-estimate follows from a standard bootstrap argument.

Lemma 2.2. Assume that there exists $C>0$ such that $|f(x, s)| \leq C\left(1+|s|^{q}\right)$ $(q<(N+2) /(N-2))$ for every $s \geq 0$, a.e. $x \in \Omega$, and that $u$ is a sub-solution of (2.3), then $u \in L^{\infty}(\Omega)$.

Remark 2.3. Once we have proved that it is bounded, under conditions of the previous lemma, we have that any solution $u$ is continuous in $\Omega$ arguing as in [14] (see Remark 2.6 in [1] for a detailed proof). Moreover, since $\partial \Omega$ is smooth, $u \in C^{0, \alpha}(\bar{\Omega})$ for some $\alpha \in(0,1)$. 
Lemma 2.4. Assume that there exists $s_{0}$ such that $f(x, s) \leq 0$ a.e. $x \in \Omega$ and for every $s>s_{0}$. Assume also that $u$ is a sub-solution of $(2.3)$, then $u \in L^{\infty}(\Omega)$ and $\|u\|_{\infty} \leq s_{0}$.

We can prove that the problem (2.2) has solution arguing as in Lemma 3.3 of [11]. We include here a sketch of the proof in order to show how to deal with the term $m(x) \in L^{\infty}(\Omega)$.

Lemma 2.5. Assume that $g \in L^{1}(0,1)$. Then there exists a solution for $(2.2)$.

Proof. We use an approximative scheme, namely

$$
\begin{cases}-\Delta u_{n}+B_{n}\left(x, u_{n}, \nabla u_{n}\right)=\min \left\{f_{0}(x), n\right\} & \text { in } \Omega, \\ u_{n}=0 & \text { on } \partial \Omega,\end{cases}
$$

where the function $B_{n}(x, s, p)$ is given, for every $(x, s, p) \in \Omega \times \mathbb{R} \times \mathbb{R}^{N}$ and $n \in \mathbb{N}$, by

$$
\begin{aligned}
B_{n}(x, s, p)= & \frac{m(x) s^{+}|p|^{2}}{\left(\frac{1}{n}+s^{+}\right)^{\theta+1}\left(1+\frac{1}{n}|p|^{2}\right)}+ \\
& +\frac{M e^{\int_{1}^{s^{+}} g\left(t+\frac{1}{n}\right) d t} \int_{0}^{s^{+}} e^{-\int_{1}^{t} g\left(\sigma+\frac{1}{n}\right) d \sigma} d t}{1+\frac{1}{n} e^{\int_{1}^{s^{+}} g\left(t+\frac{1}{n}\right) d t} \int_{0}^{s^{+}} e^{-\int_{1}^{t} g\left(\sigma+\frac{1}{n}\right) d \sigma} d t} .
\end{aligned}
$$

Since $B_{n}(x, s, p) s \geq 0$ and $B_{n}(x, s, p) \leq\|m\|_{L^{\infty}(\Omega)} n\left(n^{\theta}+M\right)$, the existence of solution $u_{n} \in H_{0}^{1}(\Omega)$ of (2.4) is deduced from [16]. Moreover, since $B_{n}(x, s, p) \geq 0$ then $-\Delta u_{n} \leq f_{0}(x)$ and, using $[22], u_{n} \in L^{\infty}(\Omega)$ and the sequence $u_{n}$ is bounded in $L^{\infty}(\Omega)$, that is, there exists $R>0$ such that

$$
\left\|u_{n}\right\|_{L^{\infty}(\Omega)} \leq R
$$

Moreover, taking $u_{n}^{-}$as test function we obtain that $u_{n} \geq 0$. Similarly, taking $u_{n}$ as test function and using the positivity of the lower order term we get that $u_{n}$ is bounded in $H_{0}^{1}(\Omega)$. Even more, taking $\min \left\{u_{n}, \varepsilon\right\} / \varepsilon$ as test function and using Fatou Lemma as $\varepsilon \rightarrow 0$ yields that

$$
\int_{\Omega} B_{n}\left(x, u_{n}, \nabla u_{n}\right) \leq\left\|f_{0}\right\|_{1} .
$$

Therefore $u_{n}$ weakly converges to $u \in H_{0}^{1}(\Omega), \nabla u_{n} \rightarrow \nabla u$ a.e. (see $[9$, Theorem 2.1]) and using Fatou Lemma as $n \rightarrow \infty$,

$$
m(x) \frac{|\nabla u|^{2}}{u^{\theta}} \chi_{\{u>0\}} \in L^{1}(\Omega) \text { and } \Psi(u) e^{G(u)} \chi_{\{u>0\}} \in L^{1}(\Omega) .
$$

In particular, since $g$ is integrable at zero, we have that $\Psi(u) e^{G(u)}$ is bounded at zero and thus, $\Psi(u) e^{G(u)} \in L^{1}(\Omega)$.

In order to pass to the limit and to prove that $u$ is the solution of (2.2) it is essential to prove that $u>0$. In order to do that we follow the ideas in [8]. Given $\tilde{m} \geq\|m\|_{L^{\infty}(\Omega)}$ we take $e^{-\tilde{m} \int_{1}^{u_{n}} \frac{1}{t^{\theta}} d t} \phi$, with $0 \leq \phi \in C_{0}^{\infty}(\Omega)$, as test function in (2.4) and we obtain

$$
\begin{array}{r}
\int_{\Omega} e^{-\tilde{m} \int_{1}^{u_{n}} \frac{1}{t^{\theta}} d t} \nabla u_{n} \cdot \nabla \phi+\int_{\Omega}\left(B_{n}\left(x, u_{n}, \nabla u_{n}\right)-\frac{\tilde{m}}{u_{n}^{\theta}}\right) e^{-\tilde{m} \int_{1}^{u_{n}} \frac{1}{t^{\theta}} d t} \phi= \\
=\int_{\Omega} \min \left\{f_{0}(x), n\right\} e^{-\tilde{m} \int_{1}^{u_{n}} \frac{1}{t^{\theta}} d t} \phi \geq \int_{\Omega} \min \left\{f_{0}(x), 1\right\} e^{-\tilde{m} \int_{1}^{u_{n}} \frac{1}{t^{\theta}} d t} \phi .
\end{array}
$$


We can use now that for $0<s<R$

$$
\begin{array}{r}
\left(B_{n}(x, s, p)-\frac{\tilde{m}}{s^{\theta}}\right) e^{-\int_{1}^{s} \frac{1}{t^{\theta}} d t} \leq \frac{m(x)-\tilde{m}}{s^{\theta}}|p|^{2} e^{-\int_{1}^{s} \frac{1}{t^{\theta}} d t}+ \\
+M e^{\int_{1}^{s}\left(g\left(t+\frac{1}{n}\right)-\frac{\tilde{m}}{t^{\theta}}\right) d t} \int_{0}^{s} e^{-\int_{1}^{t} g\left(\sigma+\frac{1}{n}\right) d \sigma} d t \leq \\
\leq M e^{\int_{1}^{s}\left(g\left(t+\frac{1}{n}\right)-\frac{\tilde{m}}{t^{\theta}}\right) d t} \int_{0}^{s} e^{-\int_{1}^{t} g\left(\sigma+\frac{1}{n}\right) d \sigma} d t \leq \\
\leq C \int_{0}^{s} e^{-\tilde{m} \int_{1}^{t} \frac{1}{\sigma^{\theta}} d \sigma} d t .
\end{array}
$$

The last inequality is due to the fact that

$$
e^{\int_{1}^{s}\left(g\left(t+\frac{1}{n}\right)-\frac{\tilde{m}}{t^{\theta}}\right) d t}=e^{\int_{1+\frac{1}{n}}^{s+\frac{1}{n}} g(\sigma) d \sigma-\int_{1}^{s} \frac{\tilde{m}}{t^{\theta}} d t} \leq e^{\int_{1}^{R+1} g(\sigma) d \sigma+\int_{0}^{1} \frac{\tilde{\tilde{\theta}}}{t^{\theta}} d t}
$$

and

$$
\begin{array}{r}
\int_{0}^{s} e^{-\int_{1}^{t} g\left(\sigma+\frac{1}{n}\right) d \sigma} d t=\int_{0}^{s} e^{\int_{1}^{t}\left(\frac{\tilde{m}}{\sigma^{\theta}}-g\left(\sigma+\frac{1}{n}\right)\right) d \sigma} e^{-\tilde{m} \int_{1}^{t} \frac{1}{\sigma^{\theta}} d \sigma} d t \leq \\
\leq e^{\int_{1}^{R+1} \frac{\tilde{m}}{\sigma^{\theta}} d \sigma+\int_{0}^{1} g\left(\sigma+\frac{1}{n}\right) d \sigma} \int_{0}^{s} e^{-\tilde{m} \int_{1}^{t} \frac{1}{\sigma^{\theta}} d \sigma} d t \leq \\
\leq e^{\int_{1}^{R+1} \frac{\tilde{m}}{\sigma^{\theta}} d \sigma+\int_{0}^{2} g(\sigma) d \sigma} \int_{0}^{s} e^{-\tilde{m} \int_{1}^{t} \frac{1}{\sigma^{\theta}} d \sigma} d t .
\end{array}
$$

Thus, we can take $C=M e^{\int_{1}^{R+1} g(\sigma) d \sigma+\int_{0}^{1} \frac{\tilde{m}}{t^{\theta}} d t} e^{\int_{1}^{R+1} \frac{\tilde{m}}{\sigma^{\theta}} d \sigma+\int_{0}^{2} g(\sigma) d \sigma}$, using (2.6) in (2.5) and denoting $\tilde{\Psi}(s)=\int_{0}^{s} e^{-\tilde{m} \int_{1}^{t} \frac{1}{\sigma^{\theta}} d \sigma} d t$ we get

$$
\int_{\Omega} \nabla \tilde{\Psi}\left(u_{n}\right) \cdot \nabla \phi+\tilde{C} \int_{\Omega} \tilde{\Psi}\left(u_{n}\right) \phi \geq \int_{\Omega} \min \left\{f_{0}(x), 1\right\} e^{-\tilde{m} \int_{1}^{u_{n}} \frac{1}{t^{\theta}} d t} \phi .
$$

From now on the proof deals exactly as in [11]. Passing to the limit in the previous inequality it follows that

$$
\int_{\Omega} \nabla \tilde{\Psi}(u) \cdot \nabla \phi+\tilde{C} \int_{\Omega} \tilde{\Psi}(u) \phi \geq \int_{\Omega} \min \left\{f_{0}(x), 1\right\} e^{-\tilde{m} \int_{1}^{u} \frac{1}{t^{\theta}} d t} \phi .
$$

Thus, the strong maximum principle allows us to assure that $0<\tilde{\Psi}(u) \leq$ $e^{\int_{0}^{1} \frac{\tilde{m}}{\sigma^{\theta}} d \sigma} u$, in particular $u>0$, and we can to pass to the limit in the approximated problem to deduce that $u \in H_{0}^{1}(\Omega)$ is a solution of (2.2) arguing as in $[8]$.

Respect to the uniqueness we prove below a comparison principle for this equation that assures that this solution is unique. This comparison principle is one of the keystones of the proof of our method. In the case $M=0$ it correspond to the comparison principle in [3, Corollary 3.5]. We include here, for the convenience of the reader, the proof of that result with the new term $M \Psi(u) e^{G(u)}$ at the left-hand side of the equation, is that to say, we prove a comparison principle for the problem (2.2) although the proof follows with no significant change that of Theorem 1.1 in $\underline{\underline{[3]} .}$

Proposition 2.6. Assume that $0<\theta<1$ and $0<m(x) \in L^{\infty}(\Omega)$. Let $\underline{u}, \bar{u} \in$ $C(\bar{\Omega})$ be, respectively, a sub and a super-solution of (2.2). Suppose also that $g \in$ 
$C^{1}(0,+\infty), e^{-G(t)} \in L^{1}(0,1), g \geq 0$ and there exists $\tau \geq 0$ such that for a.e. $x \in \Omega$ and for every $0<s<\max \left\{\|\underline{u}\|_{L^{\infty}(\Omega)},\|\bar{u}\|_{L^{\infty}(\Omega)}\right\}$ we have

$$
\tau\left[\left(-g^{\prime}(s)-\frac{m(x) \theta}{s^{\theta+1}}\right)+\left(-g(s)+\frac{m(x)}{s^{\theta}}\right) g(s)\right] \geq\left(-g(s)+\frac{m(x)}{s^{\theta}}\right)^{2} .
$$

Then $\underline{u} \leq \bar{u}$.

Proof. We will use the usual function $G_{\varepsilon}(s)=(s-\varepsilon)^{+}$for every $s \in \mathbb{R}$. We also define $w=\Psi(\underline{u})-\Psi(\bar{u})$ and observe that $G_{\varepsilon}(w)$ is bounded and has compact support in $\Omega$. In particular, $e^{-G(\underline{u})}, e^{-G(\bar{u})}, g(\underline{u}), g(\bar{u})$ are bounded in the support of $G_{\varepsilon}(w)$. Thus, for $n$ equal to the integer part of $\tau+1$, we can take $e^{-G(\underline{u})} G_{\varepsilon}(w)^{n}$ as test function in the inequality satisfied by $\underline{u}$ and $e^{-G(\bar{u})} G_{\varepsilon}(w)^{n}$ in the inequality satisfied by $\bar{u}$. Subtracting we have

$$
\begin{aligned}
0 \geq & \int_{\Omega}\left(-g(\underline{u})+\frac{m(x)}{\underline{u}^{\theta}}\right) e^{-G(\underline{u})}|\nabla \underline{u}|^{2} G_{\varepsilon}(w)^{n}- \\
& -\int_{\Omega}\left(-g(\bar{u})+\frac{m(x)}{\bar{u}^{\theta}}\right) e^{-G(\bar{u})}|\nabla \bar{u}|^{2} G_{\varepsilon}(w)^{n}+ \\
& +n \int_{\Omega} G_{\varepsilon}(w)^{n-1}\left(e^{-G(\underline{u})} \nabla \underline{u}-e^{-G(\bar{u})} \nabla \bar{u}\right) \cdot \nabla w,
\end{aligned}
$$

where we have used that

$$
\int_{\Omega}(\Psi(\underline{u})-\Psi(\bar{u})) G_{\varepsilon}(w)^{n} \geq 0 .
$$

We denote $s=\Psi^{-1}(t \Psi(\underline{u})+(1-t) \Psi(\bar{u}))$ and $\xi=t \nabla \Psi(\underline{u})+(1-t) \nabla \Psi(\bar{u})$, this means that

$$
\begin{aligned}
0 \geq & \int_{\{w>\varepsilon\}} G_{\varepsilon}(w)^{n} \int_{0}^{1} \frac{d}{d t}\left(\left(-g(s)+\frac{m(x)}{s^{\theta}}\right) e^{G(s)}|\xi|^{2}\right) d t+ \\
& +n \int_{\{w>\varepsilon\}} G_{\varepsilon}(w)^{n-1}|\nabla w|^{2} .
\end{aligned}
$$

Now we perform the derivative and we get

$$
\begin{aligned}
0 \geq & \int_{\{w>\varepsilon\}} w G_{\varepsilon}(w)^{n} \int_{0}^{1}\left(\left(-g^{\prime}(s)-\frac{m(x) \theta}{s^{\theta+1}}\right) e^{2 G(s)}|\xi|^{2}\right) d t+ \\
& +\int_{\{w>\varepsilon\}} w G_{\varepsilon}(w)^{n} \int_{0}^{1}\left(-g(s)+\frac{m(x)}{s^{\theta}}\right) g(s) e^{2 G(s)}|\xi|^{2} d t+ \\
& +\int_{\{w>\varepsilon\}} G_{\varepsilon}(w)^{n} \int_{0}^{1}\left(-g(s)+\frac{m(x)}{s^{\theta}}\right) e^{G(s)} 2 \xi \cdot \nabla w d t+ \\
& +n \int_{\{w>\varepsilon\}} G_{\varepsilon}(w)^{n-1}|\nabla w|^{2} .
\end{aligned}
$$

Multiplying by $\frac{\tau}{n}$ and taking into account that, by Young's inequality,

$$
\begin{aligned}
\frac{\tau}{n} \mid G_{\varepsilon}(w)^{n}(- & \left.g(s)+\frac{m(x)}{s^{\theta}}\right) e^{G(s)} 2 \xi \cdot \nabla w \mid \leq \\
& \leq \frac{\tau^{2}}{n} G_{\varepsilon}(w)^{n-1}|\nabla w|^{2}+\frac{G_{\varepsilon}(w)^{n+1}}{n}\left(-g(s)+\frac{m(x)}{s^{\theta}}\right)^{2} e^{2 G(s)}|\xi|^{2}
\end{aligned}
$$


it follows that

$$
\begin{aligned}
0 \geq & \left(1-\frac{\tau}{n}\right) \int_{\{w>\varepsilon\}} G_{\varepsilon}(w)^{n-1}|\nabla w|^{2}+ \\
& +\int_{\{w>\varepsilon\}} \int_{0}^{1} \frac{w G_{\varepsilon}(w)^{n} \tau e^{2 G(s)}}{n}\left[\left(-g^{\prime}(s)-\frac{m(x) \theta}{s^{\theta+1}}\right)|\xi|^{2}+\right. \\
& \left.+\left(-g(s)+\frac{m(x)}{s^{\theta}}\right) g(s)|\xi|^{2}-\frac{G_{\varepsilon}(w)}{\tau w}\left(-g(s)+\frac{m(x)}{s^{\theta}}\right)^{2}|\xi|^{2}\right] d t \geq 0 .
\end{aligned}
$$

The last inequality due to the fact that $G_{\varepsilon}(w) / w \leq 1, M \geq 0$ and (2.7). We deduce that the integrands are zero, which implies that $G_{\varepsilon}(w)=0$ for every $\varepsilon>0$, i.e., $w^{+} \equiv 0$, concluding the proof.

The following technical result plays an essential role in the further work, and it was proved in [3, Corollary 3.5] (see condition (3.6) of that paper).

Lemma 2.7. Fix $m \in L^{\infty}(\Omega), m>0$ in $\Omega$ and $\nu>0$. Then, there exist $g \in$ $C^{1}(0, \infty) \cap L^{1}(0,1)^{1}$ and $\tau$ such that if $\underline{u}$ and $\bar{u}$ are a sub and a supersolution of (2.2) such that $\max \left\{\|\underline{u}\|_{L^{\infty}(\Omega)},\|\bar{u}\|_{L^{\infty}(\Omega)}\right\} \leq \nu, g$ satisfies condition (2.7), and as consequence

$$
\underline{u} \leq \bar{u}
$$

In fact, $g$ depends on $\|m\|_{L^{\infty}(\Omega)}$ and $\theta$, but neither $\nu$ nor $\tau$. Specifically, fixed $d, C, m_{1}$ with $0<\theta<d<1, C>0$ and

$$
m_{1} \leq \min \left\{d C, C\left(\frac{d-\theta}{1-\theta}\right)^{1-\theta}\right\},
$$

for any $m \in L^{\infty}(\Omega)$ with $\|m\|_{\infty} \leq m_{1}$ we can choose $g(s) \equiv g_{\theta, d, C}(s)$ given by

$$
g_{\theta, d, C}(s)= \begin{cases}\frac{d C}{s^{\theta}}, & s<\left(\frac{\theta}{C}\right)^{\frac{1}{1-\theta}} \\ \frac{d \theta}{\theta s+\left(\frac{\theta}{C}\right)^{\frac{1}{1-\theta}}(1-\theta)}, & s \geq\left(\frac{\theta}{C}\right)^{\frac{1}{1-\theta}}\end{cases}
$$

for every $s>0$. Moreover, $\tau$ is such that

$$
\tau>\max \left\{\frac{d C+m_{1}}{C(1-d)}, \frac{2 d\left(m_{1}^{2} \nu^{2(1-\theta)}+\theta^{2}\right)}{(1-d) \theta^{2}}, \frac{2 m_{1}^{2}\left(\frac{1-\theta}{d-\theta}\right)^{2(1-\theta)}+2 d^{2} C^{2}}{d(1-d) C^{2}\left(1-\frac{m_{1}}{C}\left(\frac{1-\theta}{d-\theta}\right)^{1-\theta}\right)}\right\} .
$$

\section{The SUB-SUPERSOlution METHOD}

Now, we are ready to state the method of sub and super-solutions in order to get existence of solution of (1.1). In view of the results of the previous section the concept of sub and super-solution for (1.1) is the following.

Definition 3.1. A pair $(\underline{u}, \bar{u}),(\underline{v}, \bar{v})$ is a sub-supersolution of $(1.1)$ if $\underline{u}, \bar{u}, \underline{v}, \bar{v} \in$ $H^{1}(\Omega) \cap C(\bar{\Omega}), \underline{u}, \underline{v} \in H_{0}^{1}(\Omega)$ such that

(1) $0<\underline{u} \leq \bar{u}, 0<\underline{v} \leq \bar{v}$ almost everywhere in $\Omega$,

(2) $\frac{|\nabla \underline{u}|^{2}}{\underline{u}^{\alpha}}, \frac{|\nabla \bar{u}|^{2}}{\bar{u}^{\alpha}}, \frac{|\bar{\nabla} \underline{v}|^{2}}{\underline{v}^{\gamma}}, \frac{|\nabla \bar{v}|^{2}}{\bar{v}^{\gamma}} \in L^{1}(\Omega)$,

\footnotetext{
$1_{\text {in particular } e^{-G(t)} \in L^{1}(0,1)}$
} 
(3) for every $\phi \in H_{0}^{1}(\Omega) \cap L^{\infty}(\Omega), \phi>0$,

$$
\begin{aligned}
& \int_{\Omega} \nabla \underline{u} \cdot \nabla \phi+\int_{\Omega} g_{1}(v) \frac{|\nabla \underline{u}|^{2}}{\underline{u}^{\alpha}} \phi-\int_{\Omega} f_{1}(x, \underline{u}, v) \phi \leq 0 \leq \\
& \leq \int_{\Omega} \nabla \bar{u} \cdot \nabla \phi+\int_{\Omega} g_{1}(v) \frac{|\nabla \bar{u}|^{2}}{\bar{u}^{\alpha}} \phi-\int_{\Omega} f_{1}(x, \bar{u}, v) \phi \quad \forall v \in[\underline{v}, \bar{v}],
\end{aligned}
$$

(4) for every $\phi \in H_{0}^{1}(\Omega) \cap L^{\infty}(\Omega), \phi>0$,

$$
\begin{aligned}
& \int_{\Omega} \nabla \underline{v} \cdot \nabla \phi+\int_{\Omega} g_{2}(u) \frac{|\nabla \underline{v}|^{2}}{\underline{v}^{\gamma}} \phi-\int_{\Omega} f_{2}(x, u, \underline{v}) \phi \leq 0 \leq \\
& \leq \int_{\Omega} \nabla \bar{v} \cdot \nabla \phi+\int_{\Omega} g_{2}(u) \frac{|\nabla \bar{v}|^{2}}{\bar{v}^{\gamma}} \phi-\int_{\Omega} f_{2}(x, u, \bar{v}) \phi \quad \forall u \in[\underline{u}, \bar{u}] .
\end{aligned}
$$

Remark 3.2. Observe that any of the four inequalities in (3.1) and (3.2) is verified if it is satisfied in the classical sense, for instance, the first inequality in (3.1) is satisfied if $\underline{u}$ is twice differentiable and

$$
-\Delta \underline{u}+g_{1}(v) \frac{|\nabla \underline{u}|^{2}}{\underline{u}^{\alpha}}-f_{1}(x, \underline{u}, v) \leq 0 \text { a.e. } x \in \Omega, \forall v \in[\underline{v}, \bar{v}] .
$$

Theorem 3.3. Assume that $(\underline{u}, \bar{u}),(\underline{v}, \bar{v})$ is pair of sub-supersolution of (1.1) and denote $I:=[\underline{u}, \bar{u}] \times[\underline{v}, \bar{v}] \subset C(\bar{\Omega}) \times C(\bar{\Omega})$. Assume also the following conditions on $f_{1}, f_{2}, g_{1}$ and $g_{2}$ :

(F) There exists a constant $M \geq 0$ such that the maps $s \mapsto f_{1}(x, s, v)+M s$ and $r \mapsto f_{2}(x, u, r)+M r$ are positive and increasing for $(s, r) \in\left[0, \sup _{\bar{\Omega}} \bar{u}\right] \times$ $\left[0, \sup _{\bar{\Omega}} \bar{v}\right]$ and for all $(u, v) \in I$.

(G) $g_{1}, g_{2}$ are non-negative functions and $g_{i}(s)=0$ if and only if $s=0$ for $i=1,2$.

Then, there exists a solution $(u, v)$ of (1.1) such that $(u, v) \in I$.

Proof. Taking into account $(\mathrm{G})$, there exits a positive number $0<m_{1}$ such that

$$
0<g_{1}(z(x)), g_{2}(w(x)) \leq m_{1}, \forall(w, z) \in I .
$$

Then, taking $\nu=\max \left\{\|\bar{v}\|_{L^{\infty}(\Omega)},\|\bar{u}\|_{L^{\infty}(\Omega)}\right\}$, by Lemma 2.7 there exist $h_{1}, h_{2} \in$ $C^{1}(0,+\infty) \cap L^{1}(0,1)$ and $\tau_{1}, \tau_{2} \geq 0$ such that for a.e. $x \in \Omega$, for every $0<s<\nu$ and for every $(w, z) \in I$ we have

$$
\begin{array}{r}
\tau_{1}\left[\left(-h_{1}^{\prime}(s)-\frac{g_{1}(z(x)) \alpha}{s^{\alpha+1}}\right)+\left(-h_{1}(s)+\frac{g_{1}(z(x))}{s^{\alpha}}\right) h_{1}(s)\right] \geq \\
\geq\left(-h_{1}(s)+\frac{g_{1}(z(x))}{s^{\alpha}}\right)^{2}
\end{array}
$$

and

$$
\begin{array}{r}
\tau_{2}\left[\left(-h_{2}^{\prime}(s)-\frac{g_{2}(w(x)) \gamma}{s^{\gamma+1}}\right)+\left(-h_{2}(s)+\frac{g_{2}(w(x))}{s^{\gamma}}\right) h_{2}(s)\right] \geq \\
\geq\left(-h_{2}(s)+\frac{g_{2}(w(x))}{s^{\gamma}}\right)^{2} .
\end{array}
$$

We would like to remark again that neither $h_{i}$ nor $\tau_{i}$ depend on $(w, z)$, see Lemma 2.7 . 
Now we define

$$
G_{i}(s):=\int_{0}^{s} h_{i}(t) d t, \quad \Psi_{i}(s):=\int_{0}^{s} e^{-G_{i}(t)} d t, s>0, i=1,2 .
$$

On the other hand, observe that, using $(F)$, we get that the maps

$$
s \mapsto f_{1}(x, s, v)+M \Psi_{1}(s) e^{G_{1}(s)}, r \mapsto f_{2}(x, u, r)+M \Psi_{2}(r) e^{G_{2}(r)}
$$

are increasing for $(s, r) \in\left[0, \sup _{\bar{\Omega}} \bar{u}\right] \times\left[0, \sup _{\bar{\Omega}} \bar{v}\right]$ for all $(u, v) \in I$.

We define the operator

$$
T: I \mapsto C(\bar{\Omega}) \times C(\bar{\Omega}),(w, z) \mapsto(u, v):=T(w, z)
$$

where $(u, v) \in H_{0}^{1}(\Omega) \times H_{0}^{1}(\Omega)$ are solutions of

$$
\left\{\begin{array}{l}
-\Delta u+g_{1}(z(x)) \frac{|\nabla u|^{2}}{u^{\alpha}}+M \Psi_{1}(u) e^{G_{1}(u)}=F_{1}(x), \\
-\Delta v+g_{2}(w(x)) \frac{|\nabla v|^{2}}{v^{\gamma}}+M \Psi_{2}(v) e^{G_{2}(v)}=F_{2}(x),
\end{array}\right.
$$

with

and

$$
F_{1}(x):=f_{1}(x, w, z)+M \Psi_{1}(w) e^{G_{1}(w)}
$$

$$
F_{2}(x):=f_{2}(x, w, z)+M \Psi_{2}(z) e^{G_{2}(z)} .
$$

Taking into account $(F)$, we have that $F_{1}, F_{2}>0$. Moreover, $F_{1}, F_{2} \in L^{\frac{2 N}{N+2}}(\Omega)$, in fact, $F_{1}, F_{2} \in L^{\infty}(\Omega)$. Hence, the existence of $u$ and $v$ can be obtained from Lemma 2.5 while the uniqueness from Proposition 2.6. Therefore $T$ is well defined.

Now, we prove that $T(I) \subset I$. Indeed, take $(w, z) \in I$ and consider $(u, v)=$ $T(w, z)$, i.e. the unique solution to the problem (3.3). We are going to show that $(u, v) \in I$.

Indeed, using the definition of sub-super solution, it follows that

$$
\begin{aligned}
& -\Delta u+g_{1}(z) \frac{|\nabla u|^{2}}{u^{\alpha}}+M \Psi_{1}(u) e^{G_{1}(u)}=f_{1}(x, w, z)+M \Psi_{1}(w) e^{G_{1}(w)} \geq \\
& \geq f_{1}(x, \underline{u}, z)+M \Psi_{1}(\underline{u}) e^{G_{1}(\underline{u})} \geq-\Delta \underline{u}+g_{1}(z) \frac{|\nabla \underline{u}|^{2}}{\underline{u}^{\alpha}}+M \Psi_{1}(\underline{u}) e^{G_{1}(\underline{u})} .
\end{aligned}
$$

Proposition 2.6 allows to assure that $u \geq \underline{u}$. Analogously $u \leq \bar{u}$ and $\underline{v} \leq v \leq \bar{v}$.

Next we show that $T$ maps bounded sets into relatively compact sets. Indeed, given a bounded sequence $\left(w_{n}, z_{n}\right)$ in $I$ and denoting by $\left(u_{n}, v_{n}\right)=T\left(w_{n}, z_{n}\right)$ we have that $u_{n}$ and $v_{n}$ are bounded in $H_{0}^{1}(\Omega)$. Even more, regularity arguments (see $\underline{[22]}$ and [14]) show that sequences $u_{n}, v_{n}$ are also bounded in $C^{0, \alpha}(\bar{\Omega})$ and the compact embedding of $C^{0, \alpha}(\bar{\Omega})$ in $C(\bar{\Omega})$ implies that $u_{n} \rightarrow u$ and $v_{n} \rightarrow v$ strongly in $C(\bar{\Omega})$.

Finally, in order to have that $T$ is compact, we prove that $T$ is continuous. In order to do that, we claim that if $\left(w_{n}, z_{n}\right) \rightarrow(w, z)$ strongly in $I$ then $(u, v)=$ $T(w, z)$ (observe that this in particular implies that not only a subsequence but the whole sequence $\left(u_{n}, v_{n}\right)$ strongly converges to $T(w, z)$ in $\left.C(\bar{\Omega}) \times C(\bar{\Omega})\right)$.

In order to prove the claim we observe that

$$
\begin{array}{r}
\int_{\Omega} \nabla u_{n} \cdot \nabla \phi+\int_{\Omega} g_{1}\left(z_{n}\right) \frac{\left|\nabla u_{n}\right|^{2}}{u_{n}^{\alpha}} \phi+M \int_{\Omega} \Psi_{1}\left(u_{n}\right) e^{G_{1}\left(u_{n}\right)} \phi= \\
=\int_{\Omega} f_{1}\left(x, w_{n}, z_{n}\right) \phi+M \int_{\Omega} \Psi_{1}\left(w_{n}\right) e^{G_{1}\left(w_{n}\right)} \phi
\end{array}
$$


for every $\phi \in H_{0}^{1}(\Omega) \cap L^{\infty}(\Omega)$. We recall that, as in the proof of Lemma 2.5, the lower order term is bounded in $L^{1}(\Omega)$ and we may assume that $u_{n} \rightarrow u$ weakly in $H_{0}^{1}(\Omega)$, strongly in $L^{p}(\Omega)\left(p<2^{*}\right), u_{n}(x) \rightarrow u(x)$ and $\nabla u_{n}(x) \rightarrow \nabla u(x)$ a.e. $x \in \Omega$. Even more, since $u_{n} \geq \underline{u}$ we have that $u \geq \underline{u}>0$. In order to pass to the limit, arguing as in [8], we first consider $\phi \geq 0$ and using Lebesgue theorem as well as the weak convergence

$$
\begin{aligned}
\int_{\Omega} g_{1}\left(z_{n}\right) \frac{\left|\nabla u_{n}\right|^{2}}{u_{n}^{\alpha}} \phi \rightarrow \int_{\Omega} & f_{1}(x, w, z) \phi+M \int_{\Omega} \Psi_{1}(w) e^{G_{1}(w)} \phi- \\
& -\int_{\Omega} \nabla u \cdot \nabla \phi-M \int_{\Omega} \Psi_{1}(u) e^{G_{1}(u)} \phi .
\end{aligned}
$$

Thus, using Fatou lemma

$$
\begin{aligned}
& \int_{\Omega} g_{1}(z) \frac{|\nabla u|^{2}}{u^{\alpha}} \phi \leq \int_{\Omega} f_{1}(x, w, z) \phi+M \int_{\Omega} \Psi_{1}(w) e^{G_{1}(w)} \phi- \\
&-\int_{\Omega} \nabla u \cdot \nabla \phi-M \int_{\Omega} \Psi_{1}(u) e^{G_{1}(u)} \phi .
\end{aligned}
$$

Now we prove the reverse inequality. We take $\tilde{m}>0$ such that $\tilde{m} \geq g_{1}(z)$ for every $z \in[\underline{v}, \bar{v}]$. We consider $\phi$ with compact support, therefore we can take $e^{\int_{u_{n}}^{u} \frac{\tilde{m}}{s^{\alpha}} d s} \phi$ as test function in the equation satisfied by $u_{n}$ and we get

$$
\begin{array}{r}
\int_{\Omega} e^{\int_{u_{n}}^{u} \frac{\tilde{m}}{s^{\alpha}} d s} \nabla u_{n} \cdot \nabla \phi+\tilde{m} \int_{\Omega} e^{\int_{u_{n}}^{u} \frac{\tilde{m}}{s^{\alpha}} d s} \phi \frac{\nabla u}{u^{\alpha}} \cdot \nabla u_{n}+ \\
+\int_{\Omega}\left(M \Psi_{1}\left(u_{n}\right) e^{G_{1}\left(u_{n}\right)}-f_{1}\left(x, w_{n}, z_{n}\right)-M \Psi_{1}\left(w_{n}\right) e^{G_{1}\left(w_{n}\right)}\right) e^{\int_{u_{n}}^{u} \frac{\tilde{m}}{s^{\alpha}} d s} \phi= \\
=\int_{\Omega} \frac{\tilde{m}-g_{1}\left(z_{n}\right)}{u_{n}^{\alpha}}\left|\nabla u_{n}\right|^{2} e^{\int_{u_{n}}^{u} \frac{\tilde{m}}{s^{\alpha}} d s} \phi .
\end{array}
$$

We can pass to the limit in the left hand side of the previous inequality (using Lebesgue theorem and the weak convergence) and then Fatou Lemma in the right hand side assures that

$$
\begin{array}{r}
\int_{\Omega} \nabla u \cdot \nabla \phi+\tilde{m} \int_{\Omega} \phi \frac{|\nabla u|^{2}}{u^{\alpha}}+ \\
+\int_{\Omega}\left(M \Psi_{1}(u) e^{G_{1}(u)}-f_{1}(x, w, z)-M \Psi_{1}(w) e^{G_{1}(w)}\right) \phi \geq \\
\geq \int_{\Omega} \frac{\tilde{m}-g_{1}(z)}{u^{\alpha}}|\nabla u|^{2} \phi
\end{array}
$$

or equivalently

$$
\begin{array}{r}
\int_{\Omega} \nabla u \cdot \nabla \phi+\int_{\Omega} g_{1}(z) \frac{|\nabla u|^{2}}{u^{\alpha}} \phi+M \int \Psi_{1}(u) e^{G_{1}(u)} \phi \geq \\
\geq \int_{\Omega}\left(f_{1}(x, w, z)+M \Psi_{1}(w) e^{G_{1}(w)}\right) \phi .
\end{array}
$$

From both inequalities it yields that

$$
\begin{array}{r}
\int_{\Omega} \nabla u \cdot \nabla \phi+\int_{\Omega} g_{1}(z) \frac{|\nabla u|^{2}}{u^{\alpha}} \phi+M \int \Psi_{1}(u) e^{G_{1}(u)} \phi= \\
=\int_{\Omega}\left(f_{1}(x, w, z)+M \Psi_{1}(w) e^{G_{1}(w)}\right) \phi
\end{array}
$$


for every $\phi \in H_{0}^{1}(\Omega) \cap C_{c}(\Omega)$ and by density for every $\phi \in H_{0}^{1}(\Omega) \cap L^{\infty}(\Omega)$. Analogously $v_{n} \rightarrow v$ with $(u, v)=T(w, z)$.

Therefore, applying the Schauder Fixed Point Theorem we conclude the proof.

\section{Applications}

In this section we apply the sub-supersolution method to different systems. For that, we need some previous results.

Given $q \in L^{\infty}(\Omega)$, we denote by $\lambda_{1}(q)$ the principal eigenvalue of

$$
\begin{cases}-\Delta u+q(x) u=\lambda u & \text { in } \Omega, \\ u=0 & \text { on } \partial \Omega .\end{cases}
$$

Recall that the map $q \mapsto \lambda_{1}(q)$ is increasing. For simplicity, we denote by $\lambda_{1}:=$ $\lambda_{1}(0)$.

First consider the classical logistic equation

$$
\begin{cases}-\Delta u=u(\lambda+m(x)-u) & \text { in } \Omega, \\ u=0 & \text { on } \partial \Omega .\end{cases}
$$

It is well known that there exists a unique positive solution of (4.1) if and only if $\lambda>\lambda_{1}(-m)$. If we denote the unique positive solution by $\theta_{\lambda+m}$ we have that

$$
\theta_{\lambda+m} \leq \lambda+m_{M},
$$

where we have denoted $m_{M}=\sup _{\Omega} m$.

Now consider the logistic equation with a singular term and natural growth

$$
\begin{cases}-\Delta u+k \frac{|\nabla u|^{2}}{u^{\alpha}}=u(\lambda+m(x)-u) & \text { in } \Omega, \\ u=0 & \text { on } \partial \Omega,\end{cases}
$$

where $k>0,0<\alpha<1, \lambda \in \mathbb{R}$ and $m \in L^{\infty}(\Omega)$.

Equation (4.3) was studied in $\underline{[1]}$ when $m \equiv 0$. We prove now the following result.

Theorem 4.1. Any weak solution $u$ of (4.3) is bounded, in fact, $u \in C^{0, \alpha}(\bar{\Omega})$ and

$$
u \leq \theta_{\lambda+m} \quad \text { in } \Omega \text {. }
$$

Moreover, there exists a positive solution of (4.3) if and only if

$$
\lambda>\lambda_{1}(-m) .
$$

Furthermore, in this case there exists a maximal positive solution of (4.3), denoted by $\Theta_{[\lambda+m, \alpha, k]}$.

Finally, $\lambda+m \mapsto \Theta_{[\lambda+m, \alpha, k]}$ is increasing and $k \rightarrow \Theta_{[\lambda+m, \alpha, k]}$ is decreasing.

Proof. It is clear that if $u$ is a solution of (4.3), then $u$ is bounded. So, it is a bounded subsolution of (4.1) and hence

$$
u \leq \theta_{\lambda+m} .
$$

Assume that $\lambda \leq \lambda_{1}(-m)$ and that there exists a positive solution of (4.3). Then multiplying (4.3) by $\varphi_{1}$, a positive eigenfunction associated to $\lambda_{1}(-m)$, we get a contradiction. 
Suppose from now on that $\lambda>\lambda_{1}(-m)$. We apply the sub-supersolution method from [11] to prove the existence of a positive solution. Let us remark that although Theorem 3.5 (the sub-supersolution method) in [11] was proved for functions $f(\lambda, u)$, it is easily extended for functions $f(x, u)$.

Take

$$
(\underline{u}, \bar{u})=\left(\varepsilon \varphi_{1}^{a}, \theta_{\lambda+m}\right)
$$

where $\varepsilon>0, a>0$ and $\varphi_{1}$ is a positive eigenfunction associated to $\lambda_{1}(-m)$, that is, it satisfies

$$
\begin{cases}-\Delta \varphi_{1}-m(x) \varphi_{1}=\lambda_{1}(-m) \varphi_{1} & \text { in } \Omega \\ \varphi_{1}=0 & \text { on } \partial \Omega .\end{cases}
$$

First, we recall that $|\nabla \bar{u}|^{2} / \bar{u}^{\alpha} \in L^{1}(\Omega)$, see for instance Lemma 2.5 in [11].

On the other hand, observe that

$$
\begin{array}{r}
\nabla\left(\varphi_{1}^{a}\right)=a \varphi_{1}^{a-1} \nabla \varphi_{1}, \\
\Delta\left(\varphi_{1}^{a}\right)=a\left((a-1) \varphi_{1}^{a-2}\left|\nabla \varphi_{1}\right|^{2}+\varphi_{1}^{a}\left(-m(x)-\lambda_{1}(-m)\right)\right) .
\end{array}
$$

Hence, $|\nabla \underline{u}|^{2} / \underline{u}^{\alpha} \in L^{1}(\Omega)$ if $a>1 /(2-\alpha)$.

Since $\lambda>\lambda_{1}(-m)$, we can choose $a>1$ and $0<\eta<\lambda-\lambda_{1}(-m)$ such that

$$
\lambda>\lambda_{1}(-m) a+(a-1) m(x)+\eta .
$$

Then, $\underline{u}$ is subsolution of (4.3) if

$$
a \varphi_{1}^{-2}\left|\nabla \varphi_{1}\right|^{2}\left((1-a)+k a \varepsilon^{1-\alpha} \varphi_{1}^{a(1-\alpha)}\right)+\varepsilon \varphi_{1}^{a} \leq \lambda-\lambda_{1}(-m) a-(a-1) m(x) .
$$

Observe that the above inequality is true if

$$
k a \varepsilon^{1-\alpha} \varphi_{1}^{a(1-\alpha)} \leq a-1 \text { and } \varepsilon \varphi_{1}^{a} \leq \eta,
$$

i.e., taking $\varepsilon$ small enough. Finally, by the strong maximum principle we have that $\partial \theta_{\lambda+m} / \partial n<0$ on $\partial \Omega$, where $n$ is the outer unit normal. Thus, taking $\varepsilon$ small $\varepsilon \varphi_{1}^{a} \leq \theta_{\lambda+m}$ and so there exists a positive solution of (4.3) for $\lambda>\lambda_{1}(-m)$.

We go a little further now. We want to prove the existence of maximal solution of (4.3). Define the sequence $u^{0}=\bar{u}$ and for $n \geq 1$

$\begin{cases}-\Delta u^{n}+k \frac{\left|\nabla u^{n}\right|^{2}}{\left(u^{n}\right)^{\alpha}}+M \Psi\left(u^{n}\right) e^{G\left(u^{n}\right)}=f\left(x, u^{n-1}\right)+M \Psi\left(u^{n-1}\right) e^{G\left(u^{n-1}\right)} & \text { in } \Omega, \\ u^{n}=0 & \text { on } \partial \Omega,\end{cases}$

where $G(u)=\int_{1}^{u} g(s) d s, \Psi(s):=\int_{0}^{s} e^{-G(t)} d t$, with $g(u)=k u^{-\alpha}$, and $M \geq 0$ is such that $f(x, s)+M s$ is nondecreasing for a.e. $x \in \Omega$, where

$$
f(x, s)=s(\lambda+m(x)-s), \quad s \in\left[0, \sup _{\bar{\Omega}} \bar{u}\right] .
$$

Then, $\left\{u^{n}\right\}$ is well-defined, and by Comparison Principle (see Lemma 3.2 in [11]) we have that

$$
\underline{u} \leq \ldots \leq u^{n+1} \leq u^{n} \leq \ldots \leq u^{1} \leq u^{0}=\bar{u} .
$$

Indeed, it is easy to show that $\bar{u}$ is supersolution of the equation of $u^{1}$, and then $u^{1} \leq \bar{u}$. Assume now that $\underline{u} \leq u^{n} \leq u^{n-1}$. Then, $u^{n}$ is supersolution of the equation that verifies $u^{n+1}$, and $\underline{u}$ is subsolution, and so $\underline{u} \leq u^{n+1} \leq u^{n}$.

Moreover, we can show that

$$
u^{n} \rightarrow u^{*} \quad \text { in } H_{0}^{1}(\Omega),
$$


with $u^{*}$ a positive solution of (4.3). We claim that $u^{*}$ is the maximal solution. Indeed, take any positive solution $u$ of (4.3). We know that $u \leq \theta_{\lambda+m}=\bar{u}$, and then we can take as $\underline{u}=u$ in the above reasoning. Then, by (4.5) we obtain that

$$
u \leq u^{*}
$$

This shows the claim.

Assume that $\mu_{1}+m_{1} \leq \mu_{2}+m_{2}$ and $\mu_{1}>\lambda_{1}\left(-m_{1}\right)$. Then, it is clear that $\mu_{2}>\lambda_{1}\left(-m_{2}\right)$. Moreover, $\Theta_{\left[\mu_{1}+m_{1}, \alpha, k\right]}$ is sub-solution of (4.3) for $\lambda+m=\mu_{2}+m_{2}$ and $\bar{u}=K, K>0$ is a supersolution for large $K$. Hence, there exists a solution $u$ of (4.3) for $\lambda+m=\mu_{2}+m_{2}$ such that

$$
\Theta_{\left[\mu_{1}+m_{1}, \alpha, k\right]} \leq u \leq K,
$$

and so, since $\Theta_{\left[\mu_{2}+m_{2}, \alpha, k\right]}$ is the maximal solution, it follows that

$$
\Theta_{\left[\mu_{1}+m_{1}, \alpha, k\right]} \leq \Theta_{\left[\mu_{2}+m_{2}, \alpha, k\right]} .
$$

Using the same ideas it is easy to prove that the map $k \rightarrow \Theta_{[\lambda+m, \alpha, k]}$ is decreasing.

Remark 4.2. (1) Observe that when $k=0, \Theta_{[\lambda+m, \alpha, 0]}=\theta_{\lambda+m}$.

(2) Observe that the existence result of (4.3) does not depend on the value of $k>0$.

4.1. Example 1. We consider the system

$$
\begin{cases}-\Delta u+g_{1}(v) \frac{|\nabla u|^{2}}{u^{\alpha}}=\lambda u^{q} v^{p} & \text { in } \Omega \\ -\Delta v+g_{2}(u) \frac{|\nabla v|^{2}}{v^{\gamma}}=\mu u^{m} v^{n} & \text { in } \Omega \\ u=v=0 & \text { on } \partial \Omega\end{cases}
$$

where $\lambda, \mu \in \mathbb{R}, p, q, m, n \geq 0$, and $g_{1}$ and $g_{2}$ verify $(\mathrm{G})$. The existence results depend on the size of $p+q$ and $m+n$ and the increase and decrease of the functions $g_{1}$ and $g_{2}$.

First, it is clear that if $\lambda \leq 0$ or $\mu \leq 0$, by the maximum principle (4.6) does not have positive solution. So, we assume that $\lambda, \mu>0$. It is clear that in this case $f_{1}(x, u, v)=\lambda u^{q} v^{p}$ and $f_{2}(x, u, v)=\mu u^{m} v^{n}$ verify $(F)$ for any pair of subsupersolution of (4.6).

Theorem 4.3. Assume that $p+q<1$ and $m+n<1$. Then, there exists a positive solution if and only if $\lambda, \mu>0$.

Proof. Take

$$
(\bar{u}, \bar{v})=(K e, K e), \quad(\underline{u}, \underline{v})=\left(\varepsilon \varphi_{1}^{a}, \varepsilon \varphi_{1}^{a}\right),
$$

where $e$ is the unique positive solution of

$$
\begin{cases}-\Delta e=1 & \text { in } \Omega, \\ e=0 & \text { on } \partial \Omega,\end{cases}
$$

$\varphi_{1}$ is a positive principal eigenfunction associated to $\lambda_{1}, K$ and $\varepsilon$ are positive constants chosen large and small enough, respectively, and $a>1$. Indeed, item (1) of Definition 3.1 is trivially satisfied. Moreover, item (2) is a direct consequence of 
Lema 2.5 in [11] taking $a>\frac{1}{2-\alpha}$. With respect to items (3) and (4) we observe that for every $v \in[\underline{v}, \bar{v}]$

$$
\begin{array}{r}
-\Delta \bar{u}+g_{1}(v) \frac{|\nabla \bar{u}|^{2}}{\bar{u}^{\alpha}}-f_{1}(x, \bar{u}, v)=K+g_{1}(v) K^{2-\alpha} \frac{|\nabla e|^{2}}{e^{\alpha}}-\lambda K^{q} e^{q} v^{p} \geq \\
\geq K+g_{1}(v) K^{2-\alpha} \frac{|\nabla e|^{2}}{e^{\alpha}}-\lambda K^{p+q} e^{p+q} \geq 0 .
\end{array}
$$

The last inequality is due to $g_{1} \geq 0, p+q<1$ and that we can choose

$$
K^{1-p-q} \geq \lambda\|e\|_{\infty}^{p+q} \text {. }
$$

Similarly, in order to get

$$
0 \leq-\Delta \bar{v}+g_{2}(u) \frac{|\nabla \bar{v}|^{2}}{\bar{v}^{\gamma}}-f_{2}(x, u, \bar{v}) \phi \quad \forall u \in[\underline{u}, \bar{u}],
$$

it is sufficient that

$$
K^{1-m-n} \geq \mu\|e\|_{\infty}^{m+n}
$$

On the other hand,

$$
\begin{array}{r}
-\Delta \underline{u}+g_{1}(v) \frac{|\nabla \underline{u}|^{2}}{\underline{u}^{\alpha}}-f_{1}(x, \underline{u}, v) \phi= \\
=-\operatorname{div}\left(a \varepsilon \varphi_{1}^{a-1} \nabla \varphi_{1}\right)+g_{1}(v) a^{2} \varepsilon^{2-\alpha} \varphi_{1}^{2(a-1)-a \alpha}\left|\nabla \varphi_{1}\right|^{2}-\lambda \varepsilon^{q} \varphi_{1}^{a q} v^{p} \leq \\
\leq \lambda_{1} a \varepsilon \varphi_{1}^{a}+a(1-a) \varepsilon \varphi_{1}^{a-2}\left|\nabla \varphi_{1}\right|^{2}+g_{1}(v) a^{2} \varepsilon^{2-\alpha} \varphi_{1}^{2(a-1)-a \alpha}\left|\nabla \varphi_{1}\right|^{2}-\lambda \varepsilon^{q+p} \varphi_{1}^{a q+a p} .
\end{array}
$$

Extracting $\varepsilon^{q+p} \varphi_{1}^{a(p+q)}$ we obtain that this quantity is negative if

$$
\begin{array}{r}
\lambda_{1} a \varepsilon^{1-(p+q)} \varphi_{1}^{a(1-(p+q))}+ \\
+a \varepsilon^{1-(p+q)} \varphi_{1}^{a(1-(p+q))-2}\left|\nabla \varphi_{1}\right|^{2}\left(1-a+g_{1}(v) a \varepsilon^{1-\alpha} \varphi_{1}^{a(1-\alpha)}\right)-\lambda \leq 0
\end{array}
$$

for all $v \in[\underline{v}, \bar{v}]$. But, since $g_{1}$ in continuous in $\left[0, \sup _{\bar{\Omega}} \bar{v}\right]$, it follows the existence of a positive constant $R(\mu)$ such that $g_{1}(v) \leq R(\mu)$ for all $v \in[\underline{v}, \bar{v}]$. Thus, we can choose $\varepsilon$ such that

$$
1-a+g_{1}(v) a \varepsilon^{1-\alpha} \varphi_{1}^{a(1-\alpha)}<0 \text { and } \lambda_{1} a \varepsilon^{1-(p+q)} \varphi_{1}^{a(1-(p+q))}-\lambda<0 .
$$

Moreover, we can take $\varepsilon$ small and $K$ large such that $\underline{u} \leq \bar{u}$. Similarly, we can argue with $\underline{v}, \bar{v}$.

Theorem 4.4. Assume that $p+q<1$ and $1 \leq m+n<2-\gamma$.

(1) Assume that $g_{2}$ is increasing, $g_{2}(0)>0$. Then, for each $\lambda>0$ there exists $K(\lambda)>0$ such that if $\lambda>0$ and $\mu>K(\lambda)$, system (4.6) possesses at least a positive solution.

(2) Assume that $g_{2}$ is decreasing. Then, there exists $K>0$ such that if $\lambda>0$ and $\mu>K$ system (4.6) possesses at least a positive solution.

Proof. With the same notation of the previous proof we take on both cases

$$
(\bar{u}, \bar{v})=\left(K_{1} e, K_{2} E\right), \quad(\underline{u}, \underline{v})=\left(\varepsilon \varphi_{1}^{a}, \varphi_{1}^{a}\right),
$$

where $E(x)=\sigma \cdot x+b$ for some $\sigma \in \mathbb{R}^{N}$ with $|\sigma|>0$ and $b>0$ such that $E(x)>\eta>0$ for every $x \in \Omega$. Thus, items (1) and (2) of Definition 3.1 are 
satisfied. In order to verify the second inequality in (3.1) and in (3.2) it is enough to take $K_{1}^{1-p-q} \geq \lambda\|e\|_{\infty}^{p+q}$ and

$$
K_{2}(-\Delta E)+g_{2}(u) K_{2}^{2-\gamma} \frac{|\nabla E|^{2}}{E^{\gamma}} \geq \mu K_{2}^{m+n} e^{m} E^{n}, \quad \forall u \in[\underline{u}, \bar{u}],
$$

for which it suffices that

$$
g_{2}(u) K_{2}^{2-\gamma-m-n}|\sigma|^{2} \geq \mu\|e\|_{\infty}^{m}\|E\|_{\infty}^{n+\gamma}, \quad \forall u \in[\underline{u}, \bar{u}] .
$$

If $g_{2}$ is increasing, then $g_{2}(u) \geq g_{2}(0)$ and so we need that $2-\gamma>m+n$ and $g_{2}(0)>0$. Thus, in this case, $K_{1}$ and $K_{2}$ depend only on $\lambda$ and $\mu$, respectively.

However, if $g_{2}$ is decreasing then $g_{2}(u) \geq g_{2}\left(K_{1}\|e\|_{\infty}\right)>0$ and, using that $2-\gamma>m+n$ we can choose $K_{2}$ depending on $K_{1}$ (which depends on $\lambda$ ) and $\mu$.

On the other hand, the first inequality in (3.1) is satisfied if $\varepsilon$ is small enough and (3.2) is satisfied if

$$
\begin{array}{r}
a \varphi_{1}^{a(1-(m+n))-2}\left|\nabla \varphi_{1}\right|^{2}\left[(1-a)+g_{2}(u) a \varphi_{1}^{a(1-\alpha)}\right]+\varphi_{1}^{1-(m+n)} \leq \mu, \\
\forall u \in[\underline{u}, \bar{u}] .
\end{array}
$$

If $g_{2}$ is increasing, then $g_{2}(u) \leq g_{2}\left(K_{1}(\lambda) e\right) \leq K_{3}(\lambda)$, and so the above inequality is true for $\mu>K(\lambda)$ for some constant $K(\lambda)>0$.

If $g_{2}$ is decreasing, then $g_{2}(u) \leq g_{2}(0)$ and so the above inequality is true for $\mu$ large and independent of $\lambda$. This completes the proof.

Remark 4.5. (1) Similar result for the case $2-\alpha>p+q \geq 1$ and $m+n<1$.

(2) We could obtain results for any positive function $g_{2}$ imposing more restrictive conditions in $\lambda$ and $\mu$.

Using similar arguments of the proofs of the above results, we can show the following result.

Theorem 4.6. Assume that $1 \leq p+q<2-\alpha$ and $1 \leq m+n<2-\gamma$.

(1) Assume that $g_{1}$ and $g_{2}$ are increasing and $g_{1}(0), g_{2}(0)>0$. Then, there exist $K_{1}(\mu)$ and $K_{2}(\lambda)$ such that if $\lambda>K_{1}(\mu)$ and $\mu>K_{2}(\lambda)$ system (4.6) possesses at least a positive solution.

(2) Assume that $g_{1}$ is increasing, $g_{2}$ decreasing and $g_{1}(0)>0$. Then, there exist $K_{1}(\mu)$ and $K_{2}$ such that if $\lambda>K_{1}(\mu)$ and $\mu>K_{2}$, system (4.6) possesses at least a positive solution.

(3) Assume that $g_{1}$ and $g_{2}$ are decreasing. Then, there exist $K_{1}, K_{2}>0$ such that if $\lambda>K_{1}$ and $\mu>K_{2}$, system (4.6) possesses at least a positive solution.

4.2. Example 2: competition Lotka-Volterra system. We consider the system

$$
\begin{cases}-\Delta u+g_{1}(v) \frac{|\nabla u|^{2}}{u^{\alpha}}=u(\lambda-u-b v) & \text { in } \Omega \\ -\Delta v+g_{2}(u) \frac{|\nabla v|^{2}}{v^{\gamma}}=v(\mu-v-c u) & \text { in } \Omega \\ u=v=0 & \text { on } \partial \Omega,\end{cases}
$$

where $\lambda, \mu \in \mathbb{R}, b, c \geq 0$, and $g_{1}$ and $g_{2}$ verify $(\mathrm{G})$. When $g_{1} \equiv g_{2} \equiv 0$ system (4.7) is the classical competition Lotka-Volterra model, studied extensively in the last

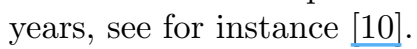


First, it is clear that if $\lambda \leq \lambda_{1}$ or $\mu \leq \lambda_{1}$ then (4.7) does not have positive solution. So, assume that $\lambda, \mu>\lambda_{1}$. Again, it is clear that $f_{1}(x, u, v)=u(\lambda-u-b v)$ and $f_{2}(x, u, v)=v(\mu-v-c u)$ verify $(F)$ for any pair of sub-supersolution of (4.7).

Theorem 4.7. Assume that one of the following conditions holds:

(1) $g_{1}$ and $g_{2}$ are increasing and $(\lambda, \mu)$ satisfies

$$
\lambda>\lambda_{1}\left(b \Theta_{\left[\mu, \gamma, g_{2}(0)\right]}\right) \quad \text { and } \quad \mu>\lambda_{1}\left(c \Theta_{\left[\lambda, \alpha, g_{1}(0)\right]}\right) ;
$$

(2) $g_{1}$ and $g_{2}$ are decreasing and $(\lambda, \mu)$ satisfies

$$
\lambda>\lambda_{1}\left(b \Theta_{\left[\mu, \gamma, g_{2}(\lambda)\right]}\right) \text { and } \mu>\lambda_{1}\left(c \Theta_{\left[\lambda, \alpha, g_{1}(\mu)\right]}\right) ;
$$

(3) $g_{1}$ is increasing, $g_{2}$ is decreasing and $(\lambda, \mu)$ satisfies

$$
\lambda>\lambda_{1}\left(b \Theta_{\left[\mu, \gamma, g_{2}(0)\right]}\right) \quad \text { and } \quad \mu>\lambda_{1}\left(c \Theta_{\left[\lambda, \alpha, g_{1}(\mu)\right]}\right) .
$$

Then (4.7) possesses a least a positive solution.

Remark 4.8. Observe that conditions (4.8), (4.9) and (4.10) define regions in the plane $(\lambda, \mu)$ which could eventually be empty. For the semilinear case, that is $g_{1} \equiv g_{2} \equiv 0$, it can be shown, see for example [18] and [17], that these regions are not empty, imposing some conditions ( $b$ or $c$ small). The study of these regions are out of the scope of this paper, but let us remark some aspects. Observe that the map

$$
\lambda \in\left[\lambda_{1}, \infty\right) \mapsto \lambda_{1}\left(c \Theta_{\left[\lambda, \alpha, g_{1}(0)\right]}\right)
$$

is increasing. Hence, for example, the region defined by (4.8) in not empty if $b$ or $c$ is small.

Proof. (1) Assume that $g_{1}$ and $g_{2}$ are increasing. Then, take

$$
\begin{aligned}
& (\bar{u}, \bar{v})=\left(\Theta_{\left[\lambda, \alpha, g_{1}(0)\right]}, \Theta_{\left[\mu, \gamma, g_{2}(0)\right]}\right), \\
& (\underline{u}, \underline{v})=\left(\Theta_{\left[\lambda-b \Theta_{\left[\mu, \gamma, g_{2}(0)\right]}, \alpha, R\right]}, \Theta_{\left[\mu-c \Theta_{\left[\lambda, \alpha, g_{1}(0)\right]}, \gamma, S\right]}\right),
\end{aligned}
$$

for some positive constants $R$ and $S$ to be chosen. Since $\underline{u}, \underline{v}, \bar{u}$ and $\bar{v}$ are solutions of logistic equations as (4.3), then items (1) and (2) of Definition 3.1 are satisfied.

Using the equation of $\bar{u}$, it can be shown that $\bar{u}$ satisfies the second inequality in (3.1) if

$$
\bar{u}(\lambda-\bar{u})-g_{1}(0) \frac{|\nabla \bar{u}|^{2}}{|\bar{u}|^{\alpha}} \geq \bar{u}(\lambda-\bar{u}-b \underline{v})-g_{1}(\underline{v}) \frac{|\nabla \bar{u}|^{2}}{|\bar{u}|^{\alpha}},
$$

or equivalently,

$$
b \bar{u} \underline{v}+\left(g_{1}(\underline{v})-g_{1}(0)\right) \frac{|\nabla \bar{u}|^{2}}{|\bar{u}|^{\alpha}} \geq 0,
$$

which is true because $g_{1}$ in increasing and $\underline{v}>0$.

For $\underline{u}$, we need that

$$
\frac{|\nabla \underline{u}|^{2}}{|\underline{u}|^{\alpha}}\left(g_{1}(\bar{v})-R\right) \leq 0, \quad \forall v \in[\underline{v}, \bar{v}] .
$$

Take $R \geq g_{1}(\bar{v})$.

Observe that by the increase of the map $\lambda+m \mapsto \Theta_{[\lambda+m, \alpha, k]}$, it follows that

$$
\underline{u} \leq \Theta_{[\lambda, \alpha, R]} \leq \Theta_{\left[\lambda, \alpha, g_{1}(0)\right]}=\bar{u},
$$

this last inequality because $R \geq g_{1}(0)$. 
(2) Assume that $g_{1}$ and $g_{2}$ are decreasing. Then, take

$$
\begin{aligned}
& (\bar{u}, \bar{v})=\left(\Theta_{\left[\lambda, \alpha, g_{1}(\mu)\right]}, \Theta_{\left[\mu, \gamma, g_{2}(\lambda)\right]}\right), \\
& (\underline{u}, \underline{v})=\left(\Theta_{\left[\lambda-b \Theta_{\left[\mu, \gamma, g_{2}(\lambda)\right]}, \alpha, g_{1}(0)\right]}, \Theta_{\left[\mu-c \Theta_{\left[\lambda, \alpha, g_{1}(\mu)\right]}, \gamma, g_{2}(0)\right]}\right) .
\end{aligned}
$$

Indeed, observe that, with a similar argument to the used in the first paragraph, $\bar{u}$ satisfies the second inequality in (3.1) if

$$
\bar{u}(\lambda-\bar{u})-g_{1}(\mu) \frac{|\nabla \bar{u}|^{2}}{|\bar{u}|^{\alpha}} \geq \bar{u}(\lambda-\bar{u}-b \underline{v})-g_{1}(\bar{v}) \frac{|\nabla \bar{u}|^{2}}{|\bar{u}|^{\alpha}},
$$

for what it is sufficient that

$$
g_{1}(\bar{v}) \geq g_{1}(\mu) .
$$

But, from (4.2) we have that $\bar{v} \leq \theta_{\mu} \leq \mu$, and since $g_{1}$ is decreasing, it follows that

$$
g_{1}(\bar{v}) \geq g_{1}(\mu) .
$$

With respect to $\underline{u}$, it can be proved that $\underline{u}$ satisfies the first inequality in $(3.1)$ because $g_{1}(\underline{v}) \leq g_{1}(0)$.

Again, it can shown that $\underline{u} \leq \bar{u}$.

(3) Assume that $g_{1}$ is increasing and $g_{2}$ is decreasing. Then, take in this case

$$
\begin{aligned}
(\bar{u}, \bar{v}) & =\left(\Theta_{\left[\lambda, \alpha, g_{1}(0)\right]}, \Theta_{\left[\mu, \gamma, g_{2}(\lambda)\right]}\right) \\
(\underline{u}, \underline{v}) & =\left(\Theta_{\left[\lambda-b \Theta_{\left[\mu, \gamma, g_{2}(\lambda)\right]}, \alpha, R\right]}, \Theta_{\left[\mu-c \Theta_{\left[\lambda, \alpha, g_{1}(0)\right]}, \gamma, g_{2}(0)\right]}\right) .
\end{aligned}
$$

4.3. Example 3: symbiotic Lotka-Volterra system. We consider the system

$$
\begin{cases}-\Delta u+g_{1}(v) \frac{|\nabla u|^{2}}{u^{\alpha}}=u(\lambda-u+b v) & \text { in } \Omega \\ -\Delta v+g_{2}(u) \frac{|\nabla v|^{2}}{v^{\gamma}}=v(\mu-v+c u) & \text { in } \Omega \\ u=v=0 & \text { on } \partial \Omega,\end{cases}
$$

where $\lambda, \mu \in \mathbb{R}, b, c>0, g_{1}$ and $g_{2} \operatorname{verify}(\mathrm{G})$.

Theorem 4.9. Assume that $b c<1$ and $(\lambda, \mu)$ satisfies

$$
\lambda>\lambda_{1}\left(-b \Theta_{\left[\mu, \gamma, \bar{g}_{2}\right]}\right) \text { and } \mu>\lambda_{1}\left(-c \Theta_{\left[\lambda, \alpha, \bar{g}_{1}\right]}\right),
$$

where $\bar{g}_{i}=g_{i}(0)$ when $g_{i}$ is decreasing and

$$
\bar{g}_{1}=g_{1}\left(\frac{\mu+c \lambda}{1-b c}\right) \quad \text { when } g_{1} \text { is increasing }
$$

and

$$
\bar{g}_{2}=g_{2}\left(\frac{\lambda+b \mu}{1-b c}\right) \text { when } g_{2} \text { is increasing. }
$$

Then (4.11) possesses a least a positive solution.

Proof. First, recall that $\Theta_{\left[\mu, \gamma, \bar{g}_{2}\right]} \leq \mu$, and then if $\lambda$ and $\mu$ verify (4.12), we have that

$$
\lambda>\lambda_{1}\left(-b \Theta_{\left[\mu, \gamma, \bar{g}_{2}\right]}\right) \geq \lambda_{1}(-b \mu)=\lambda_{1}-b \mu
$$

and so $\lambda+b \mu>0$. Analogously, $\mu+c \lambda>0$.

Now, take

$$
(\bar{u}, \bar{v})=(R, S)
$$


where $R$ and $S$ are large positive constants and

$$
\left.(\underline{u}, \underline{v})=\left(\Theta_{\left[\lambda+b \Theta_{\left[\mu, \gamma, \bar{g}_{2}\right]}, \alpha, \bar{g}_{1}\right]}, \Theta_{[\mu+c \Theta} \Theta_{\left[\lambda, \alpha, \bar{g}_{1}\right]}, \gamma, \bar{g}_{2}\right]\right) .
$$

Indeed, $R$ and $S$ must verify

$$
\lambda-R+b S \leq 0 \quad \text { and } \quad \mu-S+c R \leq 0 .
$$

Since $b c<1$, we can take

$$
R=\frac{\lambda+b \mu}{1-b c}, \quad S=\frac{\mu+c \lambda}{1-b c} .
$$

On the other hand, $\underline{u}$ is subsolution provided of

$$
g_{1}(v) \leq \bar{g}_{1}, \quad \forall v \in[\underline{v}, \bar{v}] .
$$

Then, if $g_{1}$ is decreasing (respectively increasing) we can take $\bar{g}_{1}=g_{1}(0)$ (respectively $\left.\bar{g}_{1}=g_{1}(\bar{v})=g_{1}\left(\frac{\mu+c \lambda}{1-b c}\right)\right)$.

Finally, observe that

$$
\underline{u}=\Theta_{\left[\lambda+b \Theta_{\left[\mu, \gamma, \bar{g}_{2}\right]}, \alpha, \bar{g}_{1}\right]} \leq \lambda+b\left(\Theta_{\left[\mu, \gamma, \bar{g}_{2}\right]}\right)_{M} \leq \lambda+b \mu \leq \frac{\lambda+b \mu}{1-b c}=\bar{u} .
$$

Remark 4.10. Observe again that condition (4.12) could define an empty region in the plane $(\lambda, \mu)$. As in Remark 4.8 we point out that the maps

$$
\lambda \in\left[\lambda_{1}, \infty\right) \mapsto \lambda_{1}\left(-c \Theta_{\left[\lambda, \alpha, g_{1}(0)\right]}\right)
$$

and

$$
\mu \in\left[\lambda_{1}, \infty\right) \mapsto \lambda_{1}\left(-b \Theta_{\left[\mu, \gamma, g_{2}(0)\right]}\right)
$$

are decreasing, and so the region defined by (4.12) is non empty when $g_{1}$ and $g_{2}$ are decreasing, see also $\underline{[12\rceil}$ for the semilinear case $g_{1} \equiv g_{2} \equiv 0$.

\section{ACKNOWLEDGEMENTS.}

Research supported by MICINN Ministerio de Ciencia e Innovación, Spain under grants MTM2012-31799 (JC and PJMA) and MTM2012-31304 (AS) and Junta de Andalucía FQM-116 (PJMA), FQM-194 (JC) and FQM-131 (AS).

\section{REFERENCES}

[1] D. Arcoya, J. Carmona, T. Leonori, P. J. Martínez-Aparicio, L. Orsina and F. Petitta, Existence and nonexistence of solutions for singular quadratic quasilinear equations, J. Differential Equations 246 (2009), no. 10, 4006-4042.

[2] D. Arcoya, J. Carmona and P. J. Martínez-Aparicio, Bifurcation for quasilinear elliptic singular BVP, Comm. Partial Differential Equations 36 (2011), no. 4, 670-692.

[3] D. Arcoya, J. Carmona and P. J. Martínez-Aparicio, Comparison principle for elliptic equations in divergence with singular lower order terms having natural growth. Preprint 2014.

[4] A. Bensoussan, L. Boccard and F. Murat, On a nonlinear partial differential equation having natural growth terms and unbounded solution. Ann. Inst. H. Poincaré Anal. Non Linéaire $\mathbf{5}$ (1988), no. 4, 347-364.

[5] L. Boccardo, F. Murat and J. P. Puel, Existence de solutions non bornées pour certaines équations quasi-linéaires. Portugal. Math. 41 (1982), 507-534.

[6] L. Boccardo, F. Murat and J. P. Puel, Existence de solutions faibles pour des équations elliptiques quasi-linéaires à croissance quadratique. In Nonlinear partial differential equations and their applications. Collège de France Seminar, Vol. IV (Paris, 1981/1982), 19-73. Res. Notes in Math. 84. Pitman, Boston, Mass.-London, 1983. 
[7] A. Bensoussan and L. Boccardo, Nonlinear Systems of Elliptic Equations with Natural Growth Conditions and Sign Conditions. Applied Mathematics and Optimization 46 (2002), 143-66.

[8] L. Boccardo, Dirichlet problems with singular and quadratic gradient lower order terms, ESAIM: Control, Optimization and the Calculus of Variations, 14 (2008) 411-426.

[9] L. Boccardo and F. Murat, Almost everywhere convergence of the gradients of solutions to elliptic and parabolic equations, Nonlinear Anal. 19 (1992) 581-597.

[10] R. S. Cantrell and C. Cosner, Spatial ecology via reaction-diffusion equations, Wiley Series in Mathematical and Computational Biology. John Wiley \& Sons, Ltd., Chichester, (2003).

[11] J. Carmona, P. J. Martínez-Aparicio and A. Suárez, Existence and non-existence of positive solutions for nonlinear elliptic singular equations with natural growth. Nonlinear Analysis $\mathbf{8 9}$ (2013), 157-169.

[12] M. Delgado, J. López-Gómez and A. Suárez, On the symbiotic Lotka-Volterra model with diffusion and transport effects, J. Differential Equations 160 (2000), no. 1, 175-262.

[13] J. Hernández, Qualitative methods for nonlinear diffusion equations, Nonlinear diffusion problems (Montecatini Terme, 1985), 47118, Lecture Notes in Math., 1224, Springer, Berlin, 1986.

[14] O. Ladyzenskaya and N. Uralt'seva, Linear and quasilinear elliptic equations; Translated by Scripta Technica. - New York, Academic Press, 1968.

[15] R. Landes, On the existence of weak solutions of perturbated systems with critical growth. J. Reine Angew. Math. 393 (1989), 21-38.

[16] J. Leray and J. L. Lions, Quelques résultats de Visik sur les problèmes elliptiques non linéaires par les méthodes de Minty-Browder, Bull. Soc. Math. France, 93 (1965), 97-107.

[17] J. López- Gómez and R. Pardo, Coexistence regions in Lotka-Volterra models with diffusion, Nonl. Anal. T.M.A. 19, 11-28 (1992).

[18] J. López- Gómez and J. C. Sabina, Coexistence states and global attractivity for some convective diffusive competing species models, Trans. A.M.S 347, 3797-3833 (1995).

[19] M. Montenegro and A. Suárez, Existence of a positive solution for a singular system, Proc. Roy. Soc. Edinburgh Sect. A 140 (2010), 435-447

[20] C. V. Pao, "Nonlinear Parabolic and Elliptic Equations", Plenum Press, New York, 1992.

[21] W. H. Ruan, One-parameter family of invariant set for nonweakly coupled nonlinear parabolic systems, J. Math. Anal. Appl., 189 (1995), 763-780.

[22] G. Stampacchia, Le problème de Dirichlet pour les équations elliptiques du second ordre à coefficients discontinus, Ann. Inst. Fourier (Grenoble), 15 (1965), 189-258.

(JC) Departamento de Matemáticas, Universidad de Almería, Ctra. Sacramento s/n, La Cañada de San Urbano, 04120 - Almería, Spain. JCarmona@ual.es

(PJMA) Departamento de Matemática Aplicada y Estadística, Universidad Politécnica de Cartagena, 30202 - Murcia, Spain. pedroj.martinez@upct.es

(AS) Departamento de Ecuaciones Diferenciales y Análisis Numérico, Facultad de Matemáticas, Calle Tarfia S/N, 41012-Sevilla, Spain. Suarez@us.es 